\title{
Estructura familiar, acontecimientos vitales estresantes y psicopatología en la adolescencia.
}

\author{
Family structure, stressful life events and \\ psychopathology in the teenager.
}

RESUMEN: El presente trabajo plantea estudiar el impacto de la experiencia de acontecimientos vitales estresantes y las manifestaciones clínicas de la población de adolescentes y jóvenes atendida en los Servicios de Salud Mental de Parla (Madrid, Área 10). Explorar cuáles son las variables de personalidad que determinan una respuesta diferencial ante los acontecimientos vitales estresantes, nos ayuda a comprender mejor los factores que hacen que los adolescentes resistan las adversidades de la vida, cuáles son los déficits de aquellos que sucumben y qué factores han de ponerse en marcha para lograr intervenciones eficaces.

PALABRAS CLAVE: adolescencia, acontecimientos vitales estresantes, genograma.
ABSTRACT: The aim of this research is the analysis of the impact of the stressful life experiences lived through the teenager clinic cases in the Parla mental health services (Area 10, Madrid). Scanning which personality variables carry out gap responses due to stressful vital events, it allow us to understand better the reasons how teenager can hold up the life adversities, which deficits are associated to who have failed, and which measures have to be set up to reach effective intervention.

KEY WORDS: adolescence, stressful life events, genogram.

\section{Introducción.}

Las circunstancias que caracterizan el entorno en el que se desarrolla un individuo y los acontecimientos que va afrontando, sobre todo en la infancia y la adolescencia, son factores importantes y la base sobre la cual se constituyen la propia identidad, el desarrollo de mecanismos de defensa, estrategias de afrontamiento y las habilidades sociales necesarias para su evolución.

En el Servicio de Salud Mental en el que desarrollamos nuestro trabajo, se aplica un protocolo en el primer contacto con el paciente, recogiendo datos sociodemográficos y otros relativos a la estructura familiar, mediante la elaboración de un genograma (representación gráfica de la composición familiar multigeneracional). Dichos protocolos, y en especial el genograma, reflejan en ocasiones acontecimientos vitales que pueden ser factores de riesgo, e influyen en el desarrollo del menor y adolescente. Este hecho nos condujo a cuestionarnos la relación existente entre variables sociodemográficas y la complejidad de dichos genogramas, con la existencia o no de patología y su gravedad. 
En relación a las variables sociodemográficas, Costello y cols. (1988), encuentran que un bajo nivel socioeconómico, convivir con un único padre y el fracaso escolar, están asociados a trastornos de conducta perturbadores. Grant y Compas (2003), consideran que una mala relación parental con los hijos mediatiza la relación entre un bajo nivel socioeconómico y síntomas psicopatológicos en niños y adolescentes. En este mismo estudio, hallaron que la presencia de acontecimientos vitales estresantes en la vida de los niños y adolescentes, correlaciona con la psicopatología. Proponen que entre ambos aspectos existe una relación dinámica y recíproca, en la que variables mediadoras, como las atribuciones y el afrontamiento, y variables moderadoras como la edad, el género, el soporte social y familiar, serían determinantes. Desarrollaron un modelo en el que distintas estrategias de afrontamiento del estrés tenían diferentes consecuencias sobre la psicopatología afectiva y conductual, siendo el afrontamiento centrado en el problema, el que suponía un menor desajuste frente a afrontamientos más evitativos o centrados en el malestar emocional.

Otros estudios se plantean la influencia de los acontecimientos estresantes en la psicopatología del adolescente. En concreto, Olsson y cols. (1999) estudiaron su vinculación con la sintomatología depresiva. Los adolescentes deprimidos presentan un mayor número de eventos vitales estresantes a lo largo de su vida, como plantean varias investigaciones previas (Windle, 1992; Sheeber y cols., 2007). Estos estudios hallaron que, en los casos donde los síntomas tendían a remitir en periodos breves de tiempo, los estresores estaban más relacionados con la vida cotidiana y el grupo de iguales, mientras que los casos con sintomatología más prolongada presentaban estresores relacionados con el entorno familiar, como conflictos familiares, progenitor ausente, desempleo o enfermedades graves de los padres o traslados de domicilio. Encontraron que no existía una relación causal de la depresión con el fallecimiento de uno de los padres, y que los estresores crónicos presentaban una mayor correlación con los síntomas que los agudos.

El afecto de los padres puede ser un factor protector para la sintomatología depresiva ante situaciones estresantes, pero esta misma sintomatología podría dificultar el acceso a estos apoyos por la pérdida de habilidades interpersonales que conlleva (por ejemplo en la capacidad para pedir y aceptar ayuda) (Sandler y cols., 1994).

En general, la tendencia actual de la investigación se orienta hacia contemplar no sólo los factores de riesgo sino también los factores de protección frente a la psicopatología; como los estilos de afrontamiento y la personalidad del propio adolescente (sentimientos de autoeficacia, locus de control interno, resiliencia, ...), el apoyo familiar (habilidades de comunicación, confianza y empatía), y otros factores como la posibilidad de encontrar referentes positivos en maestros y otros adultos de la comunidad a la que pertenece (Joseph y cols., 2007). 
En cuanto a la relación entre psicopatología en los padres y la salud mental en los hijos, hay diferentes estudios que reflejan dicha influencia. Sanz y cols. (2006), realizan un estudio en el Centro de Salud Mental de Getafe (Madrid) con una muestra de 395 pacientes del Programa de niños y adolescentes que acuden a consulta. De ellos el $41 \%$ de los mismos tienen un familiar en tratamiento, siendo la mayor parte de las veces la madre $(26 \%)$.

Fergusson y cols. (1995) encuentran correlación entre los síntomas depresivos en la madre y los de sus hijos. No ocurre con los padres. Para estos autores, esta correlación en el caso de la madre está asociada a desventajas importantes a nivel social, falta de apoyo por parte del padre, desacuerdos en la pareja y adversidad familiar. Burt y cols. (2003), demuestran que uno de los predictores más potentes de depresión y trastornos en niños y adolescentes, es tener un progenitor deprimido. Rutter y cols. (1992) constataron en una investigación que chicos con alteraciones ansiosas presentaban en mayor porcentaje padres con este tipo de trastorno.

En un estudio realizado por Bragado y cols. (1999), con una muestra de niños y adolescentes entre 6-17 años, se encuentra que tener un padre joven, estar muy preocupado por la muerte de un ser querido, discusiones y peleas familiares, haber sido maltratado físicamente, repetir curso y tener problemas de comportamiento en la infancia, se relaciona con el riesgo de sufrir trastornos de conducta. Los trastornos de ansiedad están asociados a la edad de la madre, problemas en el parto, miedo a ser maltratado, estar preocupado por la familia y tomar medicación a diario. Y por último, el tener más edad, estar preocupado por la familia, el alcoholismo familiar, las complicaciones durante el embarazo, tener enfermedades frecuentes, dificultades físicas y el estado psicopatológico de la madre, están relacionados con trastornos depresivos.

Con respecto al alcoholismo en los padres, los hijos de padres alcohólicos tienen significativamente más problemas psicopatológicos que los hijos de padres no alcohólicos (Aragón, 1997).

Además de la psicopatología de los padres, las relaciones del adolescente con su familia también han sido objeto de estudio. Desde el modelo sistémico, se proponen ciertos modelos de comunicación como explicativos de muchas alteraciones psicopatológicas, como obsesiones, trastornos de la alimentación y conductas problemáticas (Nardone y cols., 2003). Kagan (1979) en un estudio prospectivo investigó la relación entre el estilo de familia hiperprotector y la aparición en la adolescencia de cuadros ansiosos, obsesivos, fóbicos, depresivos y alteraciones de la alimentación. Igualmente, Vélez y cols. (1989) plantea que la estructura familiar y el tipo de convivencia (familias reconstruidas, familia monoparental, etc.) están asociados a trastornos de la conducta. Sin embargo, su importancia en dicho trastorno es menos relevante que otros factores de riesgo. 
Otra variable de la organización familiar que ha recibido bastante atención es el divorcio de los padres. Kasen y cols. (1996) encontraron que la situación familiar post-divorcio ejerce una notable influencia en niños adolescentes. El efecto es independiente del temperamento previo del niño y de los aspectos educacionales, pero ejerce diferente efecto en función del sexo. Los hijos varones de padres divorciados que vivían sólo con la madre presentaban un alto riesgo de sufrir depresión, mientras que este efecto no se confirmó en las mujeres. Sin embargo, en la familia reestructurada, la presencia de un padrastro se relaciona con un riesgo elevado de trastornos de ansiedad y depresión en las mujeres y no sucede así en los varones. Estudios posteriores (Laumann-Billings y Emery, 2000) matizan estos hallazgos, planteando que no hay diferencias en el BDI (Inventario de Depresión de Beck) entre hijos de padres divorciados y no divorciados, pero que sí se encuentra malestar asociado a la experiencia de divorcio, como sentimientos de pérdida, dudas del amor de los padres y la sensación de que habrían sido personas diferentes de no haber vivido esa experiencia. Plantean también que no es tanto la experiencia del divorcio en sí, sino todos los estresores que la rodean (el conflicto previo, la formación de nuevas familias, etc.) lo que influye en el malestar.

Sheeber y cols. (2007) plantearon que no es sólo la existencia de conflicto con los padres, sino también la ausencia de apoyo por parte de éstos, lo que se relaciona con la psicopatología. Sin embargo, en situaciones de alto conflicto, el apoyo no parece ser suficiente para proteger al menor de la aparición de síntomas. Igualmente, disponer de una buena relación con uno de los progenitores tampoco es suficiente para evitar el desarrollo de sintomatología depresiva si existe un grave conflicto con el otro progenitor. En este sentido hallaron diferencias entre población sana, subclínica y depresiva en los niveles de conflicto y apoyo percibidos, tanto maternos como paternos.

Un caso extremo de organización disfuncional en la familia es la del maltrato. La experiencia de maltrato puede conllevar un amplio espectro de alteraciones psicopatológicas, entre las que se incluyen depresión mayor (Stein y cols., 1996), aislamiento y una pobre relación con los compañeros (Kashani y cols., 1987), mayor índice de suicidio en la adolescencia (Kopp, 1997), y una mayor prevalencia de los trastornos de ansiedad y depresión en la vida adulta (Green, 1997).

En relación a los adolescentes y sus manifestaciones psicopatológicas, Feduchi (2006) en un estudio sobre las formas de expresión de las conductas violentas en la adolescencia, concluye que dichas conductas están relacionadas con:

- Vivencias de desatención en aspectos como: la confianza, el reconocimiento, estímulos y oportunidades.

- Episodios traumáticos en la historia del joven y la vivencia de repetición. 
ORIGINALES Y REVISIONES

- Relaciones de sobreprotección en el entorno familiar, que dificultan el proceso de individuación y autonomización del adolescente y ocultan el sentimiento de culpa en los padres.

- El mecanismo defensivo de identificación con el agresor cuando existen malos tratos en el entorno familiar y se vive a la figura maltratadora como poderosa, a la que se teme y admira a la vez de forma intensa. Existe una dificultad para tolerar la frustración en el adolescente.

La adolescencia es un momento vital donde las experiencias de pérdida y duelo están presentes y tienen una especial relevancia. Villanueva y García (2000), en un trabajo en el que profundizan en la especificidad del duelo en la infancia y adolescencia en una población de menores atendidos, en el Servicio de Salud Mental de Parla (Madrid), encuentran que el duelo en estas edades se manifiesta no tanto desde una sintomatología depresiva (16\% de los casos atendidos), sino con alteraciones de conducta (31\%) y fracaso en el rendimiento académico (20\%). Un $50 \%$ de situaciones catastróficas (en las que se suceden varias pérdidas precoces y significativas, que generan a su vez otros cambios y pérdidas y aumentan la vulnerabilidad), se producen cuando ocurren dos cambios o más (de vivienda, colegio, cuidadores, etc.), relacionados con la institucionalización, alcoholismo de la madre y enfermedad grave en los padres.

Para Siegel, (1996), el periodo terminal de la enfermedad de los padres es más estresante que la pérdida. Aparecen síntomas de ansiedad ante la enfermedad grave de los padres y somatizaciones y sintomatología depresiva ante la muerte de un familiar.

Parker, (1992), constata que las experiencias precoces de pérdidas hacen más difícil la elaboración del duelo y contribuyen al desarrollo de rasgos de personalidad suspicaz, tendencia a la rumiación, introversión, neuroticismo y conductas desadaptadas.

A partir de la investigación previa, y de la sensación general en nuestro día a día de que la población adolescente en Salud Mental presenta una historia vital compleja y con múltiples situaciones que podrían comprometer su desarrollo, en este trabajo nos proponemos constatar si realmente se da un mayor número de acontecimientos estresantes en esta población, y si correlacionan con el grado de psicopatología. Y dando un paso más allá, intentar encontrar relaciones entre eventos concretos y formas específicas de afrontamiento que lleguen a constituir los síntomas. 
Método.

\section{Muestra}

Los sujetos participantes en este estudio forman parte de la población adolescente con edades comprendidas entre 16 y 21 años, atendida en los Servicios de Salud Mental de Parla entre junio de 2007 y febrero de 2008. La muestra está compuesta por 62 sujetos con una edad media de 19,35 años (Desviación Típica=1,37).

Procedimiento

El diseño empleado en este trabajo consiste en un estudio observacional transversal. La recogida de información se estructuró en dos partes. En la primera, se recogieron las variables sociodemográficas incluidas en el impreso estándar que cumplimenta el personal administrativo con todos los pacientes que acuden al centro. Asimismo, también se recogió a partir del historial clínico, el diagnóstico CIE-9 (OMS, 1992) que era asignado a cada paciente después de las entrevistas de evaluación con algún profesional del Centro de Salud Mental (psicólogo clínico o psiquiatra).

En la segunda parte, se obtuvo la información acerca del resto de variables analizadas en el presente trabajo mediante un protocolo conformado por dos cuestionarios autoaplicados. Dicho protocolo se entregaba a los sujetos en su primer contacto con el Centro de Salud Mental para que los cumplimentaran y lo entregaran en su primera cita.

Para confeccionar el primer cuestionario, tomamos algunos de los eventos vitales que contempla el Cuestionario de experiencias vitales estresantes elaborado por Coddington (1972), ampliándolo con otros acontecimientos que nos parecían relevantes en la población que íbamos a investigar, registrando la edad en la que ocurrieron, su duración y la percepción subjetiva de su influencia, para determinar el grado de impacto de los mismos. Consta de 30 ítems de respuesta dicotómica, para establecer la frecuencia de cada evento y escalas tipo likert para medir su efecto.

El segundo cuestionario empleado fue el MACI (Inventario Clínico para Adolescentes de Millon). El MACI ha sido diseñado para evaluar características de personalidad y síndromes clínicos en adolescentes. Consta de 160 elementos que se agrupan en 27 escalas divididas en tres grandes áreas: Características de Personalidad, Preocupaciones Expresadas y Síndromes Clínicos (Millon, 2004).

Una vez recogidos los datos de autoevaluación, se procedió a la realización de los análisis a través del paquete estadístico SPSS 13.0. 
ORIGINALES Y REVISIONES

\section{Resultados.}

Frecuencia y características de la exposición a acontecimientos traumáticos.

El 98,4\% de los adolescentes participantes en el estudio había estado expuesto a una o más de las treinta experiencias vitales estresantes incluidas en el protocolo (Figura 1).

La distribución del tipo de eventos traumáticos en la muestra del estudio se recoge en la Tabla 2. Para comprobar si existían diferencias estadísticas con relación al género, se ha utilizado la prueba Chi-cuadrado. No se observaron diferencias estadísticamente significativas entre el género y los acontecimientos vitales estudiados.

Características de la estructura familiar

Todos los sujetos estudiados convivían con su familia biológica. En la Figura 2 se resumen las frecuencias de las distintas estructuras familiares encontradas, siendo la más frecuente la estructura familiar tradicional.

También se estudió la existencia de antecedentes familiares de problemas de salud mental. En este sentido, encontramos que sólo un tercio de la muestra no presentaba antecedentes familiares de ningún tipo (33,9\%). Un 40,3\% presentaban antecedentes maternos, un $11,3 \%$ antecedentes paternos y un $43,5 \%$, en otros familiares.

Distribución de los diagnósticos

La distribución de los diagnósticos clínicos de los pacientes, agrupados según los grupos diagnósticos de la CIE-9 (OMS, 1992) y el género, se encuentra recogida en la Tabla 3 (2 sujetos de la muestra tenían diagnóstico aplazado). Los trastornos más frecuentes, tanto en varones como en mujeres, fueron los adaptativos, neuróticos y reacciones de estrés que suponen la mayoría de los casos de la muestra. Aunque las diferencias no alcanzaron significación estadística $(\chi 2$ : $6,731 ; \mathrm{p}=0,151)$, en los varones resultaron más frecuentes los trastornos neuróticos (50\% frente a $20,5 \%$ en mujeres) mientras que en las mujeres lo fueron los trastornos adaptativos y las reacciones de estrés (45,5\% frente a 31,3\%). Sin embargo, en los trastornos de personalidad, la tasa no varía en función del género (18\% en los dos sexos). Por último, todos los casos de trastorno de alimentación fueron diagnosticados en el subgrupo de mujeres $(13,6 \%)$.

Análisis descriptivo y correlacional de las variables de personalidad y los factores psicopatológicos estudiados

A continuación aparece una relación general de cada uno de los factores del MACI con sus descriptivos correspondientes: (tabla 4)

La tabla 5 muestra los resultados correspondientes al análisis de correlación que se establecen entre las dimensiones de personalidad de los pacientes en su perfil del MACI y las variables psicopatológicas evaluadas por la misma prueba 
(síndromes clínicos y preocupaciones expresadas).

Del análisis de esta tabla se desprende que entre los factores de personalidad estudiados y el resto de variables psicopatológicas, destaca especialmente la correlación negativa y significativa que se establece entre la escala de Discordancia Familiar y las dimensiones de personalidad más internalizantes (inhibido, sumiso y conformista); mientras que hallamos una correlación positiva y significativa con las dimensiones de personalidad más externalizantes (rebelde, rudo, oposicionista y límite).

Análisis univariado y multivariado de la varianza

Se aplicó una prueba t para comprobar la existencia de diferencias en las variables de personalidad y psicopatológicas en función de los eventos vitales. En la tabla 6 se resumen las dimensiones para las que se han hallado diferencias significativas en cada uno de los eventos vitales estudiados.

Asimismo, con el fin de examinar la relación entre las dimensiones psicopatológicas y clínicas con la estructura de la familia de origen de los adolescentes, se llevó a cabo un análisis multivariado de la varianza (MANOVA), tomando los factores sociodemográficos y familiares como variables independientes y las dimensiones del MACI como variables dependientes. Para las dimensiones del MACI, los resultados del contraste de significación multivariada no han arrojado diferencias significativas en ninguna de las variables dependientes estudiadas.

\section{Discusión.}

Nuestro trabajo pretende relacionar acontecimientos vitales y características familiares con variables clínicas en el adolescente. A la vista de los resultados, la prevalencia de eventos vitales estresantes experimentados por los adolecentes que demandan asistencia en los Servicios de Salud Mental (98,4\% de la muestra) está muy por encima de las cifras que se manejan en los estudios con población general, que oscilan en torno al 40\% (Breslau y cols., 1991) y al 72\% (Oliva y cols, 2008).

Diversos autores han señalado la necesidad de considerar que no todos los factores estresantes tienen el mismo impacto en los adolescentes, ni responden del mismo modo ante las situaciones de estrés, existiendo una gran variabilidad individual al respecto (Antonovsky, 1987; Lazarus y Folkman, 1984; Kobasa, 1979). Las variables moduladoras que hemos estudiado en nuestro trabajo son potenciales eventos traumáticos en la historia vital de los sujetos. Tomando en cuenta los 30 acontecimientos vitales estudiados, encontramos una media de 5,95 eventos experimentados por cada adolescente (Desviación Típica $=3,26)$, llegan- 
do en algunos casos a vivenciar hasta 15 un solo individuo (tan sólo 1 sujeto de la muestra no ha experimentado ningún acontecimiento vital de los recogidos en la Escala construida para este fin).

Algunos de los acontecimientos estudiados son el conflicto entre los padres y el divorcio. Los resultados muestran una correlación con la rebeldía como variable de personalidad y con la predisposición a la delincuencia, la propensión a la impulsividad y el afecto depresivo como síndromes clínicos. Los sentimientos y la conducta de los jóvenes están relacionados con la manera inadecuada que tienen los adultos de resolver dicho conflicto, con la tensión que desencadena y con la utilización de los menores por parte de los padres, cuyo efecto más extremo sería el síndrome de alienación parental. La rebeldía en muchos casos es una respuesta adaptativa relacionada con el malestar y el descontento en los adolescentes.

La predisposición a la delincuencia y la propensión a la impulsividad pueden estar relacionadas con un entorno inestable, con falta de normas y con la pérdida de los padres como figuras de apoyo. Por otra parte, la ausencia de uno de los progenitores en el hogar dificulta la supervisión de los menores, constituyendo una variable de riesgo para las conductas disruptivas. La tristeza, la situación de ruptura, de pérdida y de duelo que se pueden experimentar cuando hay un divorcio en los padres, se manifiesta con afecto depresivo o con conductas disociales o externalizantes, más propias en esta edad.

Otro de los eventos importantes analizados es la violencia familiar, tanto física como verbal, y tanto observada como sufrida. Siempre supone un nivel de crispación y tensión en el ambiente familiar, tensión que se transmite a toda la familia y se manifiesta en los jóvenes con rebeldía como rasgo de personalidad. Este evento es muy traumático y con consecuencias importantes en el desarrollo del niño y del adolescente. Es un modelo de aprendizaje negativo como forma de abordar los desacuerdos. Encontramos tres niveles de intensidad, ser testigo de violencia verbal, que provoca sentimientos de ansiedad, ser testigo de violencia física como circunstancia más traumática, y sufrir maltrato físico cuyo efecto es más paralizante y desencadena sentimientos de indefensión (Seligman, 1991). Los dos últimos tipos de violencia se expresan con afecto depresivo.

Destacar también la incomodidad con el sexo como preocupación en los jóvenes cuando son testigos de violencia física en la familia. Supone un modelo de identificación negativo siempre, tanto si se identifica con el agresor o con el agredido, tanto si el joven es hombre o mujer y tanto si es el padre o la madre el que agrede. Implica asociar afecto y sexualidad con violencia.

En la adolescencia, la relación con el grupo de iguales o amigos constituye un pilar importante en la construcción de la identidad. En esta etapa, se busca lealtad y compromiso, pudiendo ser relaciones muy exigentes en ese sentido. 
Otro aspecto importante es la aceptación del propio cuerpo y las vivencias en relación a la sexualidad. Estas son dos áreas fundamentales y a su vez, muy frágiles en el desarrollo del adolescente. Cuando se producen cambios en la aceptación de los amigos, se generan sentimientos de inseguridad, de baja autoestima y cuestionamiento de sí mismo y de su cuerpo, por lo que en los resultados aparece la incomodidad con el sexo como preocupación y los trastornos de la alimentación como síndrome clínico.

Respecto a la enfermedad del padre o de la madre, el análisis de datos no muestra diferencias significativas en cuanto a la psicopatología si se contemplan por separado, sin embargo al contemplarlos de manera conjunta, la enfermedad de los progenitores se relaciona con desvalorización y ansiedad como síndrome clínico. Una posible explicación sería que, en estadíos tempranos del desarrollo, ver la debilidad de las figuras de referencia que tendrían que suponer una base segura, podría favorecer la aparición de la ansiedad y de sentimientos de incertidumbre. Por otra parte, la enfermedad de uno de los padres puede favorecer que los hijos vean al otro progenitor preocupado o angustiado, observando con ello un modelo de afrontamiento de este tipo. También es llamativo que aquellos individuos que durante su infancia han vivido la enfermedad de un progenitor, presenten sentimientos de desvalorización de sí mismos. El pensamiento mágico y el egocentrismo de los niños en etapas tempranas del desarrollo podría favorecer que éstos se sientan responsables del malestar que presentan los adultos y contribuir a la aparición de una baja autoestima. Vivir la enfermedad de un hermano se relaciona con la aparición de rasgos de sumisión; probablemente el estrés y la preocupación de los padres por otro de los hijos, hace que el resto intente encontrar su lugar o bien no generando problemas o bien adoptando un rol de hipermadurez, colaboración y obediencia a los padres.

En cuanto al acontecimiento vital fallecimiento del padre, se observa en los adolescentes la aparición de rasgos de sumisión, inseguridad con los iguales y tendencia al suicidio. La ausencia del padre podría provocar que la madre buscase el apoyo emocional en los hijos, dificultando su salida hacia la socialización y favoreciendo las conductas de obediencia y apoyo al progenitor. La tendencia al suicidio podría ser una forma extrema de no aceptación de la pérdida o bien relacionarse con los síntomas depresivos que algunos autores (por ejemplo; Guidano, 1994) asocian con la experiencia de duelos en la infancia. El análisis de datos no revela diferencias significativas ante la pérdida de la madre; lo cual se explica por el tamaño de la muestra (un sujeto). Tomando conjuntamente la pérdida del padre o de la madre, se observa una respuesta de sumisión en los menores, que se explicaría como hemos comentando anteriormente.

Respecto al fallecimiento de un hermano, se observa la presencia de rasgos histriónicos en la conducta de los adolescentes. Una posible explicación se 
ORIGINALES Y REVISIONES

relacionaría con el duelo de los padres y la necesidad de los hijos de llamar su atención. Este mismo rasgo aparece también ante la pérdida de un abuelo, junto con la inhibición y la propensión a la impulsividad. Nos resulta significativo que un acontecimiento vital que es esperable atendiendo al ciclo vital, tenga tantas consecuencias en el desarrollo de los adolescentes. Parece que las respuestas dadas a este acontecimiento por el adolescente pueden ser tanto internalizantes (tendencia a la inhibición) como externalizantes.

La muerte de un amigo provoca en los adolescentes de nuestra muestra, respuestas de alteración y oposicionismo. Ambas podrían interpretarse como formas de protesta ante una pérdida inesperada.

Haber estado a cargo de personas distintas de los padres dentro de la familia, se relaciona con un estilo de respuesta autopunitivo que quizá responde al sentimiento de culpa por el abandono. Por otra parte, estas cesiones son vividas a veces como impuestas por parte del adulto que se hace cargo y pueden despertar rabia hacia el progenitor que no se ocupó del menor y con el que además existe un vínculo familiar. Esto podría conllevar la verbalización de estas emociones favoreciendo la culpabilización en el niño. Sería la existencia del vínculo familiar lo que posibilita la aparición de respuestas emocionales intensas con su consecuente emoción expresada, y por ello este rasgo autopunitivo no aparecería cuando la cesión se realiza con personas ajenas a la familia. En este último caso, lo que aparece es el egocentrismo como rasgo de personalidad. Probablemente, esto se debe a que algunas de estas cesiones suponen vivir en instituciones con otros muchos menores, en las que parece importante ocuparse prioritariamente de las necesidades propias.

Algunos de los eventos vitales que consideramos importantes y creemos que repercuten en la psicopatología en los jóvenes no aparecen como significativos en nuestro estudio, como sucede en el caso del suicidio o intento de suicidio en personas cercanas a los adolescentes. En nuestra opinión, este resultado se relaciona con las limitaciones de la muestra.

De manera similar, al estudiar los acontecimientos vitales relacionados con el consumo de drogas en padres y hermanos, no aparecen diferencias significativas en el análisis estadístico. Una posible hipótesis sería que el consumo de drogas, al estar más sancionado socialmente que el de alcohol, podría ser menos visible para los adolescentes falseando nuestros resultados. El consumo de alcohol en los padres se relaciona con múltiples alteraciones en los adolescentes de la muestra: rebeldía y rasgos de personalidad límite, incomodidad con el sexo, abusos en la infancia, ansiedad como síndrome clínico y tendencia al suicidio. $\mathrm{El}$ alcoholismo de uno de los padres provoca severas alteraciones en la vida familiar que pueden ir desde las dificultades económicas a la violencia, pasando por el abuso sexual, e influyendo negativamente en la construcción de la identidad 
del adolescente. Supone además, la aparición de sentimientos de vergüenza por el estigma social asociado a este consumo y la ansiedad por la pérdida del sentimiento de seguridad en el hogar. Es llamativo que el consumo de alcohol en los hermanos no suponga diferencias significativas según el análisis de datos y esto pone de manifiesto la relevancia del déficit en el rol parental para el desarrollo de alteraciones.

Respecto al traslado de ciudad por parte de la familia encontramos distintas respuestas: inhibición, sumisión y rebeldía, que reflejan diferentes modelos de adaptación a grupos sociales nuevos. El hecho de que estas respuestas configuren rasgos de personalidad estables y no sólo reacciones de adaptación podría explicarse atendiendo al momento evolutivo en el que la familia se hubiera trasladado.

El traslado de uno de los progenitores por motivos laborales presenta también diferencias significativas en cuanto a la discordancia familiar, el abuso sexual y la tendencia al suicidio. Nos resulta llamativo el dato referente al abuso sexual, quizás la ausencia de uno de los progenitores diluye las fronteras entre el sistema parental y el filial constituyendo esto una variable de riesgo; o bien dicha ausencia dificulta la protección de los menores y además provoca que se recurra a adultos ajenos al núcleo familiar para el cuidado de los niños. En cuanto a la discordancia familiar, resulta fácil entender que la ausencia de uno de los progenitores pueda constituirse como un foco de conflicto entre los adultos.

Por último, tener que repetir un curso escolar está relacionado con pesimismo, rudeza, autopunición y propensión a la impulsividad. Se observa nuevamente los dos estilos de respuesta externalizante e internalizante, constituyendo expresiones de protesta o bien, sentimientos de culpa y depresión como formas de afrontamiento.

Explorar cuáles son las variables de personalidad que determinan una respuesta diferencial ante los acontecimientos vitales estresantes, nos ayuda a comprender mejor los factores que hacen que los adolescentes resistan las adversidades de la vida, cuáles son los déficits de aquellos que sucumben y qué factores han de ponerse en marcha para lograr intervenciones eficaces (Sánchez y cols., 2007).

Los resultados de nuestro estudio muestran que aún queda mucho por investigar sobre la influencia de los acontecimientos vitales estresantes en el desarrollo y la psicopatología de los adolescentes. Por ello, serían necesarias investigaciones más específicas que pudieran profundizar en cómo afectan cada uno de ellos, poniendo a prueba las hipótesis que planteamos en la discusión. 
Tabla 1

Características sociodemográficas de la muestra

\begin{tabular}{|c|c|c|c|}
\hline & $\%(\mathrm{n})$ & & $\%(n)$ \\
\hline Género & & Nivel de estudios de la madre & \\
\hline Hombre & $25,8(16)$ & Sin estudios & $0(0)$ \\
\hline Mujer & $74,2(46)$ & Educación primaria & $45,2(28)$ \\
\hline \multirow{5}{*}{$\begin{array}{l}\text { Edad de la primera consulta } \\
\text { De } 0 \text { a } 6 \text { años } \\
\text { De } 7 \text { a } 12 \text { años } \\
\text { De } 13 \text { a } 17 \text { años } \\
\text { De } 17 \text { a } 21 \text { años }\end{array}$} & \multirow{5}{*}{$\begin{array}{c}1,6(1) \\
8,1(5) \\
25,8(16) \\
64,5(40)\end{array}$} & Educación secundaria & $48,4(30)$ \\
\hline & & Estudios superiores & $6,5(4)$ \\
\hline & & Nivel de estudios del padre & \\
\hline & & Sin estudios & $6,5(4)$ \\
\hline & & Educación primaria & $56,5(35)$ \\
\hline \multirow{6}{*}{$\begin{array}{c}\text { Orden de la fratría } \\
1 \\
2 \\
3 \\
4 \\
5\end{array}$} & \multirow{6}{*}{$\begin{array}{c}48,4(30) \\
33,9(21) \\
11,3(7) \\
4,8(3) \\
1,6(1)\end{array}$} & Educación secundaria & $32,3(20)$ \\
\hline & & \begin{tabular}{|l} 
Estudios superiores \\
\end{tabular} & $4,8(3)$ \\
\hline & & Ocupación de la madre & \\
\hline & & Profesionales y técnicos & $8,1(5)$ \\
\hline & & Administrativo & $8,1(5)$ \\
\hline & & Comerciales y vendedores & $8,1(5)$ \\
\hline \multirow{6}{*}{$\begin{array}{l}\text { Convivencia } \\
\text { Con ambos padres } \\
\text { Monoparental } \\
\text { Con otros miembros de la familia extensa } \\
\text { Madre/padre y nueva pareja } \\
\text { Otros }\end{array}$} & \multirow{6}{*}{$\begin{array}{c}61,3(38) \\
16,1(10) \\
6,5(4) \\
9,7(6) \\
6,5(4)\end{array}$} & Hostelería, servicios y seguridad & $41,9(26)$ \\
\hline & & Industria, construcción y transportes & $8,1(5)$ \\
\hline & & Ama de casa & $14,5(9)$ \\
\hline & & Otros & $11,3(7)$ \\
\hline & & Ocupación del padre & \\
\hline & & Profesionales y técnicos & $9,7(6)$ \\
\hline \multirow{7}{*}{$\begin{array}{l}\text { Nivel de estudios } \\
\text { No escolarizado } \\
\text { Educación primaria } \\
\text { Educación secundaria } \\
\text { Bachillerato } \\
\text { Módulo primer grado } \\
\text { Módulo segundo grado }\end{array}$} & \multirow{7}{*}{$\begin{array}{c}1,6(1) \\
8,1(5) \\
35,5(22) \\
37,1(23) \\
6,5(4) \\
11,3(7)\end{array}$} & Administrativo & $0(0)$ \\
\hline & & Comerciales y vendedores & $9,7(6)$ \\
\hline & & Hostelería, servicios y seguridad & $6,5(4)$ \\
\hline & & Industria, construcción y transportes & $64,5(40)$ \\
\hline & & Amo de casa & $0(0)$ \\
\hline & & Otros & $9,7(6)$ \\
\hline & & Situación laboral de la madre & \\
\hline \multicolumn{2}{|l|}{ Escolarización } & Trabaja & $67,7(42)$ \\
\hline Adecuado para su edad & $35,5(22)$ & En paro & $4,8(3)$ \\
\hline Retrasado un año & $37,1(23)$ & Ama de casa & $25,8(16)$ \\
\hline Retrasado dos o más años & $27,4(17)$ & Otros & $1,6(1)$ \\
\hline \multicolumn{2}{|l|}{ Situación laboral } & Situación laboral del padre & \\
\hline Estudia & $53,2(33)$ & Trabaja & $80,6(50)$ \\
\hline Trabaja & $30,6(19)$ & En paro & $0(0)$ \\
\hline Parado & $9,7(6)$ & Amo de casa & $0(0)$ \\
\hline Otros & $6,5(4)$ & Otros & $19,4(12)$ \\
\hline
\end{tabular}


Tabla 2

Frecuencia de los distintos acontecimientos traumáticos.

\begin{tabular}{|c|c|c|c|c|c|c|}
\hline \multirow[b]{3}{*}{ Divorcio de los padres } & \multicolumn{2}{|c|}{$\begin{array}{c}\text { Hombres } \\
(n=16)\end{array}$} & \multicolumn{2}{|c|}{$\begin{array}{c}\text { Mujeres } \\
(n=46)\end{array}$} & \multicolumn{2}{|c|}{$\begin{array}{c}\text { Total } \\
(n=62)\end{array}$} \\
\hline & $\mathbf{N}$ & $\%$ & $\mathbf{N}$ & $\%$ & $\mathbf{N}$ & $\%$ \\
\hline & 3 & $18,8 \%$ & 14 & $30,4 \%$ & 17 & $27,4 \%$ \\
\hline Ser testigo de violencia verbal en la familia & 5 & $31,3 \%$ & 23 & $50 \%$ & 28 & $45,2 \%$ \\
\hline Ser testigo de violencia física en la familia & 3 & $18,8 \%$ & 9 & $19,6 \%$ & 12 & $19,4 \%$ \\
\hline Sufrir maltrato físico en la familia & 2 & $12,5 \%$ & 9 & $19,6 \%$ & 11 & $17,7 \%$ \\
\hline Conflicto entre los padres & 7 & $43,8 \%$ & 24 & $52,2 \%$ & 31 & $50 \%$ \\
\hline La familia se traslada de país & 0 & $0 \%$ & 1 & $2,2 \%$ & 1 & $1,6 \%$ \\
\hline La familia se traslada de ciudad & 2 & $12,5 \%$ & 13 & $28,3 \%$ & 15 & $24,2 \%$ \\
\hline Paro de uno de los padres & 2 & $12,5 \%$ & 10 & $21,7 \%$ & 12 & $19,4 \%$ \\
\hline Uno de los padres se traslada por trabajo & 3 & $18,8 \%$ & 1 & $2,2 \%$ & 4 & $6,5 \%$ \\
\hline Enfermedad de la madre & 7 & $43,8 \%$ & 10 & $21,7 \%$ & 17 & $27,4 \%$ \\
\hline Enfermedad del padre & 6 & $37,5 \%$ & 12 & $26,1 \%$ & 18 & $29 \%$ \\
\hline Enfermedad de uno de los hermanos & 1 & $6,3 \%$ & 5 & $10,9 \%$ & 6 & $9,7 \%$ \\
\hline $\begin{array}{l}\text { Haber estado a cargo de personas distintas } \\
\text { de los padres (de la familia) }\end{array}$ & 6 & $37,5 \%$ & 9 & $19,6 \%$ & 15 & $24,2 \%$ \\
\hline $\begin{array}{l}\text { Haber estado a cargo de personas distintas } \\
\text { de los padres (fuera de la familia) }\end{array}$ & 2 & $12,5 \%$ & 1 & $2,2 \%$ & 3 & $4,8 \%$ \\
\hline Consumo de drogas en los padres & 1 & $6,3 \%$ & 4 & $8,7 \%$ & 5 & $8,1 \%$ \\
\hline Consumo de drogas en los hermanos & 0 & $0 \%$ & 1 & $2,2 \%$ & 1 & $1,6 \%$ \\
\hline Consumo de alcohol en los padres & 4 & $25 \%$ & 9 & $19,6 \%$ & 13 & $21 \%$ \\
\hline Consumo de alcohol en los hermanos & 0 & $0 \%$ & 2 & $4,3 \%$ & 2 & $3,2 \%$ \\
\hline $\begin{array}{l}\text { Suicidio o intentos de suicidio en } \\
\text { personas cercanas }\end{array}$ & 2 & $12,5 \%$ & 10 & $21,7 \%$ & 12 & $19,4 \%$ \\
\hline Los hermanos se marchan de casa & 5 & $31,3 \%$ & 11 & $23,9 \%$ & 16 & $25,8 \%$ \\
\hline Cambios de aceptación en los amigos & 6 & $37,5 \%$ & 23 & $50 \%$ & 29 & $46,8 \%$ \\
\hline Uno de los padres ingresa en prisión & 0 & $0 \%$ & 2 & $4,3 \%$ & 2 & $3,2 \%$ \\
\hline Muerte de la madre & 1 & $6,3 \%$ & 0 & $0 \%$ & 1 & $1,6 \%$ \\
\hline Muerte del padre & 1 & $6,3 \%$ & 4 & $8,7 \%$ & 5 & $8,1 \%$ \\
\hline Muerte de los hermanos & 1 & $6,3 \%$ & 1 & $2,2 \%$ & 2 & $3,2 \%$ \\
\hline Muerte del abuelo/a & 11 & $68,8 \%$ & 24 & $52,2 \%$ & 35 & $56,5 \%$ \\
\hline Muerte de un amigo/a & 1 & $6,3 \%$ & 4 & $8,7 \%$ & 5 & $8,1 \%$ \\
\hline Padecer una enfermedad física & 2 & $12,5 \%$ & 7 & $15,2 \%$ & 9 & $14,5 \%$ \\
\hline Repetir un curso & 12 & $75 \%$ & 26 & $56,5 \%$ & 38 & $61,3 \%$ \\
\hline $\begin{array}{l}\text { Quedarse embarazada o dejar embarazada } \\
\text { a una chica }\end{array}$ & 1 & $6,3 \%$ & 1 & $2,2 \%$ & 2 & $3,2 \%$ \\
\hline
\end{tabular}




\section{ORIGINALES Y REVISIONES}

Tabla 3

Frecuencia de los diagnósticos en función del género

\begin{tabular}{l|c|c|c|c|c|c}
\hline \multirow{2}{*}{} & \multicolumn{2}{|c|}{$\begin{array}{c}\text { Hombres } \\
(\mathbf{n = 1 6 )}\end{array}$} & \multicolumn{2}{c|}{$\begin{array}{c}\text { Mujeres } \\
(\mathbf{n = 4 4 )}\end{array}$} & \multicolumn{2}{c}{$\begin{array}{c}\text { Total } \\
(\mathbf{n}=\mathbf{6 0})\end{array}$} \\
\cline { 2 - 7 } & $\mathbf{N}$ & $\mathbf{\%}$ & $\mathbf{N}$ & $\mathbf{\%}$ & $\mathbf{N}$ & $\mathbf{\%}$ \\
\cline { 2 - 7 } Trastornos neuróticos & 8 & 50,0 & 9 & 20,5 & 17 & 28,3 \\
\hline Trastornos adaptativos y reacciones de estré & 5 & 31,3 & 20 & 45,5 & 25 & 41,7 \\
\hline Trastornos de la alimentación & 0 & 0 & 6 & 13,6 & 6 & 10,0 \\
\hline Trastornos de la personalidad & 3 & 18,8 & 8 & 18,2 & 11 & 18,3 \\
\hline Alteraciones de la conducta & 0 & 0 & 1 & 2,2 & 1 & 1,7 \\
\hline
\end{tabular}

Tabla 4

Análisis descriptivo de los factores analizados en el MACI.

\begin{tabular}{|c|c|c|c|c|c|}
\hline \multicolumn{2}{|c|}{ Variable } & Mínimo & Máximo & Media & D.T. \\
\hline \multirow[t]{4}{*}{ Escalas psicométricas } & Transparencia & 0,00 & 92,00 & 55,73 & 22,09 \\
\hline & Deseabilidad & 0,00 & 100,00 & 61,29 & 22,38 \\
\hline & Alteración & 2,00 & 100,00 & 55,51 & 23,86 \\
\hline & Introvertido & 8,00 & 92,00 & 46,76 & 21,18 \\
\hline \multirow[t]{11}{*}{ Características de personalidad } & Inhibido & 3,00 & 90,00 & 45,24 & 22,33 \\
\hline & Pesimista & 14,00 & 87,00 & 47,52 & 18,31 \\
\hline & Sumiso & 1,00 & 109,00 & 57,48 & 22,69 \\
\hline & Histriónico & 1,00 & 104,00 & 51,61 & 27,17 \\
\hline & Egocéntrico & 1,00 & 109,00 & 52,74 & 27,59 \\
\hline & Rebelde & 1,00 & 113,00 & 47,76 & 20,96 \\
\hline & Rudo & 3,00 & 110,00 & 48,95 & 22,16 \\
\hline & Conformista & 1,00 & 115,00 & 60,69 & 25,77 \\
\hline & Oposicionista & 15,00 & 98,00 & 45,85 & 18,81 \\
\hline & Autopunitivo & 13,00 & 88,00 & 46,34 & 19,26 \\
\hline & Límite & 11,00 & 105 & 46,92 & 19,63 \\
\hline \multirow[t]{8}{*}{ Preocupaciones expresadas } & Difusión de identidad & 16,00 & 97,00 & 49,26 & 20,11 \\
\hline & Desvalorización & 12,00 & 105,00 & 53,48 & 22,40 \\
\hline & Desagrado por el cuerpo & 14,00 & 107,00 & 54,22 & 21,39 \\
\hline & Incomodidad con el sexo & 1,00 & 115,00 & 51,13 & 24,98 \\
\hline & Inseguridad con iguales & 17,00 & 115,00 & 55,47 & 22,15 \\
\hline & Insensibilidad social & 0,00 & 115,00 & 54,74 & 27,95 \\
\hline & Discordancia familiar & 16,00 & 108,00 & 49,19 & 21,36 \\
\hline & Abusos en la infancia & 6,00 & 107,00 & 52,35 & 22,57 \\
\hline \multirow[t]{7}{*}{ Síndromes clínicos } & Trastornos de la alimentación & 9,00 & 98,00 & 50,69 & 19,04 \\
\hline & Inclinación a sustancias & 1,00 & 109,00 & 51,37 & 21,38 \\
\hline & Predisposición a delincuencia & 1,00 & 112,00 & 52,64 & 22,82 \\
\hline & Propensión a impulsividad & 10,00 & 113,00 & 51,45 & 23,72 \\
\hline & Ansiedad & 1,00 & 114,00 & 54,95 & 21,29 \\
\hline & Ánimo depresivo & 10,00 & 105,00 & 53,45 & 22,14 \\
\hline & Tendencia al suicidio & 9,00 & 105,00 & 49,98 & 21,59 \\
\hline
\end{tabular}




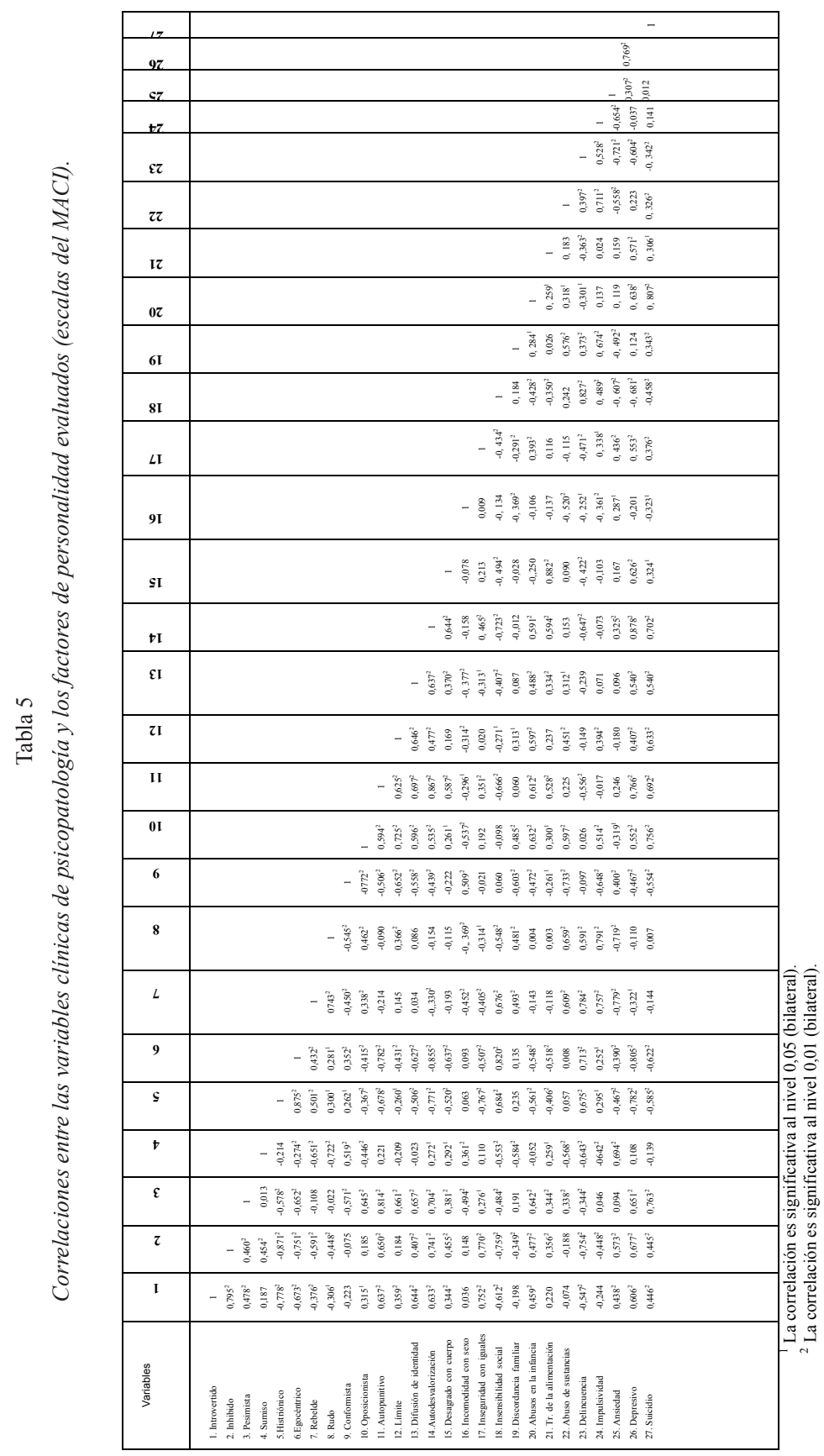




\section{ORIGINALES Y REVISIONES}

Tabla 6

Resultados de la prueba t en las dimensiones con diferencias estadísticamente significativas.

\begin{tabular}{|c|c|c|c|}
\hline Evento & Variable clínica (MACI) & $\mathbf{T}$ & $\mathbf{p}$ \\
\hline 1. Divorcio de los padres & $\begin{array}{l}\text { - Rebeldía (personalidad) } \\
\text { - } \text { Predisposición a la delincuencia (sd. clínico) } \\
\text { - Afecto depresivo (sd. clínico) }\end{array}$ & $\begin{array}{l}-0,042 \\
-0,447 \\
-0,734 \\
\end{array}$ & $\begin{array}{l}0,013 \\
0,044 \\
0,003\end{array}$ \\
\hline $\begin{array}{l}\text { 2. Ser testigo de violencia } \\
\text { verbal en la familia }\end{array}$ & $\begin{array}{l}\text { - Rebeldía (personalidad) } \\
\text { - Sentimientos de ansiedad (sd. clínico) }\end{array}$ & $\begin{array}{l}-0,408 \\
0,496\end{array}$ & $\begin{array}{l}0,012 \\
0,029\end{array}$ \\
\hline $\begin{array}{l}\text { 3. Ser testigo de violencia física } \\
\text { en la familia }\end{array}$ & $\begin{array}{l}\text { - Rebeldía (personalidad) } \\
\text { - Incomodidad con el sexo (preocup. expresada) } \\
\text { - Afecto depresivo (sd. clínico) }\end{array}$ & $\begin{array}{l}-0,029 \\
-0,236 \\
-1,218\end{array}$ & $\begin{array}{l}0,036 \\
0,030 \\
0,033\end{array}$ \\
\hline $\begin{array}{l}\text { 4. Sufrir maltrato físico en la } \\
\text { familia }\end{array}$ & - Afecto depresivo (sd. clínico) & $-0,149$ & 0,054 \\
\hline 5. Conflictos entre los padres & $\begin{array}{l}\text { - Rebeldía (personalidad) } \\
\text { - Propensión a la impulsividad (sd. clínico) }\end{array}$ & $\begin{array}{ll}0,319 \\
-0,170 \\
\end{array}$ & $\begin{array}{l}0,004 \\
0,037 \\
\end{array}$ \\
\hline 6. La familia se traslada de país & No diferencias significativas (sólo 1 sujeto) & & \\
\hline $\begin{array}{l}\text { 7. . La familia se traslada de } \\
\text { ciudad }\end{array}$ & $\begin{array}{l}\text { - Inhibido (personalidad) } \\
\text { - Sumisión (personalidad) } \\
\text { - Rebeldía (personalidad } \\
\end{array}$ & $\begin{array}{l}0,087 \\
-0,295 \\
1,111 \\
\end{array}$ & $\begin{array}{l}0,033 \\
0,041 \\
0,052\end{array}$ \\
\hline 8. Paro de uno de los padres & No diferencias significativas & & \\
\hline $\begin{array}{l}\text { 9. Uno de los padres se traslada } \\
\text { por trabajo }\end{array}$ & $\begin{array}{l}\text { - Discordancia familiar (preocup. expresada) } \\
\text { - Abusos en la infancia (preocup. expresada) } \\
\text { - Tendencia al suicidio (sd. clínico) } \\
\end{array}$ & $\begin{array}{l}0,139 \\
-1,712 \\
-1,252 \\
\end{array}$ & $\begin{array}{l}0,028 \\
0,006 \\
0,053\end{array}$ \\
\hline 10. Enfermedad de la madre & No diferencias significativas. & & \\
\hline 11. Enfermedad del padre & No diferencias significativas & & \\
\hline $\begin{array}{l}\text { 12. Enfermedad de uno de los } \\
\text { hermanos }\end{array}$ & - Sumisión (personalidad) & 0,393 & 0,034 \\
\hline $\begin{array}{l}\text { 13. Haber estado a cargo de } \\
\text { personas distintas de los padres } \\
\text { (de la familia) }\end{array}$ & - Autopunitivo (personalidad) & $-2,275$ & 0,050 \\
\hline $\begin{array}{l}\text { 14. Haber estado a cargo de } \\
\text { personas distintas de los padres } \\
\text { (fuera de la familia) }\end{array}$ & - Egocentrismo (personalidad) & 0,495 & 0,035 \\
\hline $\begin{array}{l}\text { 15. Consumo de drogas en los } \\
\text { padres }\end{array}$ & No diferencias significativas & & \\
\hline $\begin{array}{l}\text { 16. Consumo de drogas en los } \\
\text { hermanos }\end{array}$ & No diferencias, sólo un sujeto & & \\
\hline $\begin{array}{l}\text { 17. Consumo de alcohol en los } \\
\text { padres }\end{array}$ & $\begin{array}{l}\text { - Rebeldía (personalidad) } \\
\text { - Límite (personalidad) } \\
\text { - Ancomodidad con el sexo (preocup. expresada) } \\
\text { - Ansies en la infancia (preocup. expresada) } \\
\text { - Tendencia al Suicico) } \\
\end{array}$ & $\begin{array}{l}0,352 \\
-1,694 \\
0,095 \\
-3,753 \\
-0,009 \\
-2,322\end{array}$ & $\begin{array}{l}0,026 \\
0,015 \\
0,004 \\
0,008 \\
0,015 \\
0,017\end{array}$ \\
\hline $\begin{array}{l}\text { 18. Consumo de alcohol en los } \\
\text { hermanos }\end{array}$ & No diferencias significativas. & & \\
\hline $\begin{array}{l}\text { 19. Suicidios o intentos de } \\
\text { suicidio en personas cercanas }\end{array}$ & No diferencias significativas. & & \\
\hline $\begin{array}{l}\text { 20. Los hermanos se marchan } \\
\text { de casa. }\end{array}$ & No diferencias significativas. & & \\
\hline $\begin{array}{l}\text { 21. Cambios en la aceptación de } \\
\text { los amigos. }\end{array}$ & $\begin{array}{ll} & \text { Incomodidad con el sexo (preocup. expresada) } \\
- & \text { Trastorno de Conducta Alimentaria(sd. clínico) }\end{array}$ & $\begin{array}{l}1,162 \\
0,573\end{array}$ & $\begin{array}{l}0,040 \\
0,057\end{array}$ \\
\hline $\begin{array}{l}\text { 22. Uno de los padres ingresa } \\
\text { en prisión. }\end{array}$ & No diferencias significativas. & & \\
\hline 23. Muerte de la madre & No diferencias, sólo 1 sujeto. & & \\
\hline 24. Muerte del padre. & $\begin{array}{ll} & \text { Sumisión (personalidad). } \\
- & \text { Inseguridad con iguales (preocup. expresada) } \\
\text { - } & \text { Tendencia al suicidio (sd. clínico) } \\
\end{array}$ & $\begin{array}{l}-0,338 \\
0,174 \\
-0,130 \\
\end{array}$ & $\begin{array}{l}0,033 \\
0,052 \\
0,054 \\
\end{array}$ \\
\hline 25. Muerte de un/a hermano/a. & - $\quad$ Histriónico (personalidad) & $-1,107$ & 0,057 \\
\hline 26. Muerte de abuelo/a & $\begin{array}{ll} & \text { Inhibido (personalidad) } \\
\text { - } & \text { Histriónico (personalidad) } \\
& \text { Propensión a la impulsividad (sd. clínico) } \\
\end{array}$ & $\begin{array}{l}-2,066 \\
2,117 \\
0,524 \\
\end{array}$ & $\begin{array}{l}0,056 \\
0,052 \\
0,048\end{array}$ \\
\hline 27. Muerte de un/a amigo/a. & $\begin{array}{ll}\text { - } & \text { Alteración (escala modificadora) } \\
\text { - Oposicionismo (personalidad) } \\
\end{array}$ & $\begin{array}{l}0,614 \\
0,748 \\
\end{array}$ & $\begin{array}{l}0,049 \\
0,056\end{array}$ \\
\hline $\begin{array}{l}\text { 28. Padecer una enfermedad } \\
\text { fisica }\end{array}$ & No diferencias significativas. & & \\
\hline 29. Repetir un curso & $\begin{array}{ll} & \text { Pesimismo (personalidad) } \\
\text { - } & \text { Rudeza (personalidad) } \\
\text { - } & \text { Propensión án a la impulsividad (sd. clínico) } \\
\end{array}$ & $\begin{array}{l}0,178 \\
-1,397 \\
-0,405 \\
-1,695 \\
\end{array}$ & $\begin{array}{l}0,009 \\
0,037 \\
0,011 \\
0,045 \\
\end{array}$ \\
\hline $\begin{array}{l}\text { 30. Quedarse embarazada o } \\
\text { dejar embarazada a una chica. }\end{array}$ & No diferencias significativas. & & \\
\hline Pérdida progenitor & • $\quad$ Sumiso (personalidad) & 4,266 & 0,043 \\
\hline Enfermedad progenitor & $\begin{array}{ll}- & \text { Desvalorización (preocupación expresada) } \\
\text { - } & \text { Ansiedad (sd. clínico) }\end{array}$ & $\begin{array}{l}-4,603 \\
-1,199 \\
\end{array}$ & $\begin{array}{l}0,046 \\
0,032\end{array}$ \\
\hline
\end{tabular}


Figura 1

Tasa de acontecimientos traumáticos por persona.

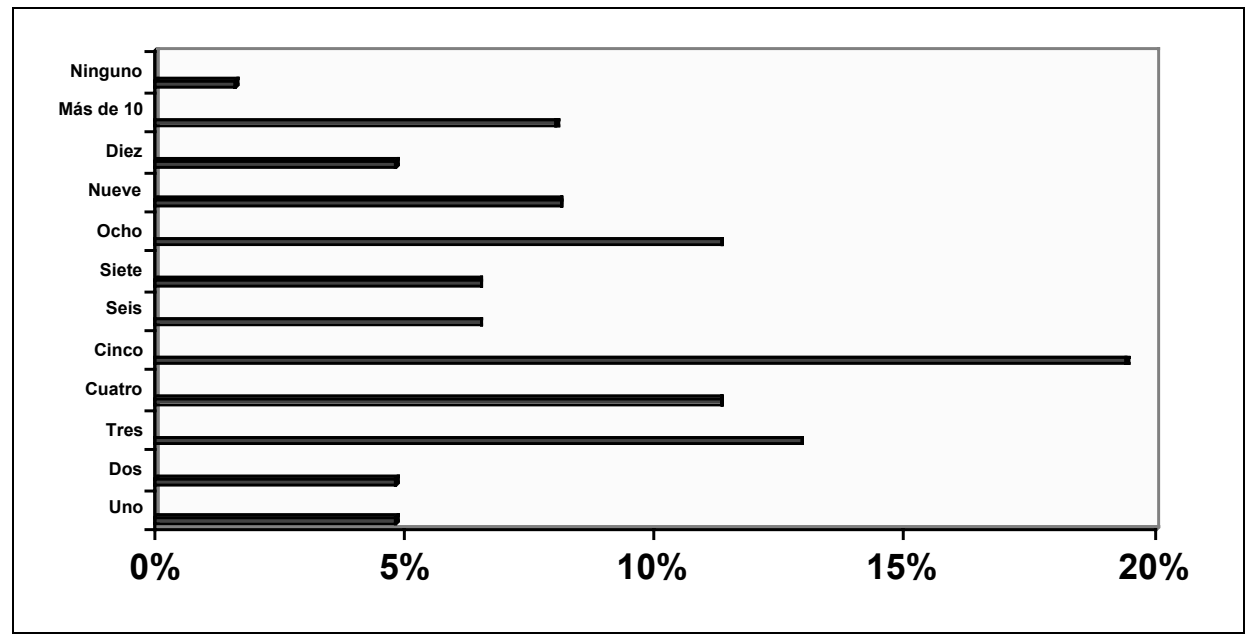

Figura 2

Frecuencia de las distintas estructuras familiares.

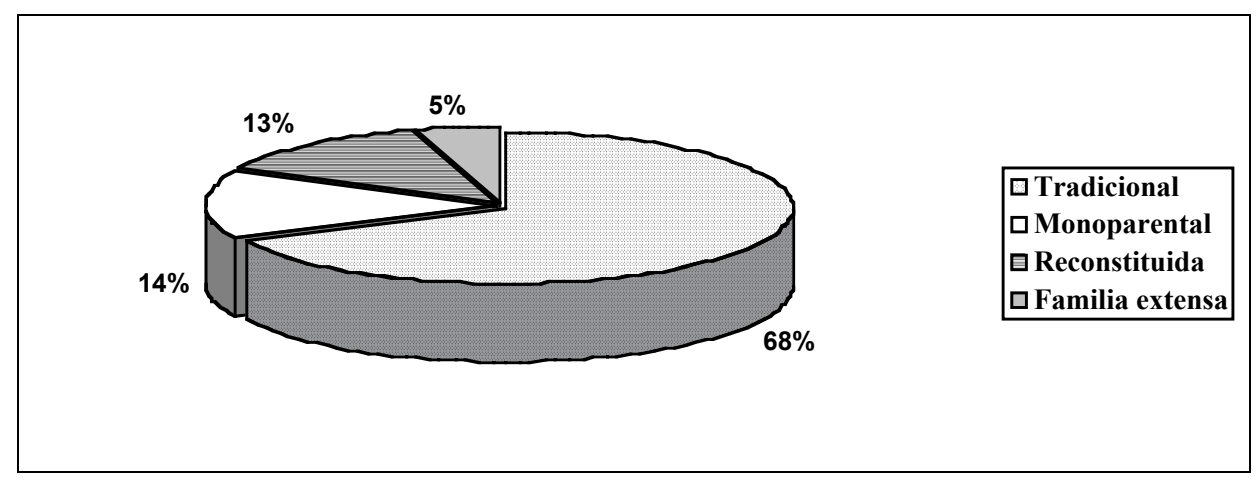


ORIGINALES Y REVISIONES

\section{BIBLIOGRAFÍA:}

(1) Antonovsky, A. (1987). Unravelling the mystery of health. How people manage stress and stay well. California: Jossey-Bass.

(2) Aragón, N.; Bragado, C.; Carrasco, I. (1997). Alcoholismo parental y psicopatología infantil. Una revisión. Adicciones, 9, 255-277.

(3) Bragado, C.; Bersabé, R.; CARRASCO, I. (1999). Factores de riesgo para los trastornos conductuales, de ansiedad, depresivos y de eliminación en niños y adolescentes. Psicothema, 11 (4), 939-956.

(4) Breslau, N.; Davis, G. C.; Andreski, P.; Petersen, E. (1991). Traumatic events and posttraumatic stress disorder in an urban population of young adults. Archives of General Psychiatry, 48: $216-222$

(5) Burt, S. A.; Krueger, R. F., Mcgue, M.; Iacono, W. (2003). Parent-child conflict and the comorbidity among childhood externalizing disorders. Archives of General Psychiatry, 60, 505-513.

(6) Coddington, R. D. (1972). The significance of life events as etiologic factors in diseases of children. A survey of professional workers. Journal of Psychosomatic Research, 62, 7-18.

(7) Costello, E. J.; Costello, A. J.; Edelbrock, C.; Burns, B.; Dulcan, M. K.; Brent, D.; JANISZEWSKI, S. (1988). Psychiatric disorders in pediatric primary care. Archives of General Psychiatry, 45, 1107-1116.

(8) Feduchi, L.; Mauri, P.; Raventós, P.; Sastre, V.; Tió, J. (2006). Reflexiones en torno a la violencia en la adolescencia. Psicopatología y salud mental del niño y del adolescente, 8, 19-26.

(9) Fergusson, D. M.; Horwood, L. J.; Lynskey, M. T. (1995). Maternal depressive symptoms and depressives symptoms in adolescents. Journal of child Psychology and Psychiatry, 36, 1161-1178.

(10) Grant, K.E.; Compas, F. E. (2003). Stressors and child and adolescent psychopathology: moving from to markers to mechanisms of risk. Psychological Bulletin, 129 (3), 447-466.

(11) Green, A. H. (1997). The impact of physical, sexual, and emotional abuse. En: J. D. Noshpitz: P. F. Kernberg y J. R. Bemporad (Eds.), Handbook of child and adolescent psychiatry 2, pp. 201-212. New York: John Wiley \& Sons.

(12) Guidano, V. (1994). El sí mismo en proceso. Barcelona: Paidos.

(13) JosePh, S.; KnibBs, J.; Hobbs, J. (2007). Trauma, Resilience, and growth in children and adolescents. En: A. A. Hosin (Ed.), Responses to traumatizad children, pp. 148-161. Basingstoke: Pulgrave McMillan.

(14) Kagan, J. (1979). Family experience and the child's development. American Psychologist, 34 (10), 886-891.

(15) Kasen, S.; Cohen, P.; Brook, J. S.; Hartmark, C. (1996). A multiple risk interaction model: effects of temperament and divorce on psychiatry disorder in children. Journal of abnormal child psychology, 24, 121-150.

(16) Kashani, J. H.; Beck, N. C.; BuRK, J. P. (1987) Predictors of psychopathology in children of patients with major affective disorders. Canadian journal of psychiatry, 32, 287-292.

(17) Kobasa, S. C. (1979). Stressful life events, personality, and health. An inquiry into hardiness. Journal of personality and Social Psychology, 31: 1-11.

(18) Kopp, C.B.; Mcintosh, J. M. (1997). High risk environments and young children. En: J. D. Noshpitz; S. Greens; S. Wieder; and J. Osofsky (Eds.), Handbook of child and adolescent psychiatry 1, pp. 160-176. New York: John Wiley \& Sons. 
(19) Laumann-Billings, L.; Emery, R. E. (2000). Distress among young adults from divorced families. Journal of family psychology, 14 (4), 671-687.

(20) Lazarus, R.S., Folkman, S. (1984). Stress, appraisal and coping. New York: Springer.

(21) Millon, T. (2004). Inventario clínico para adolescentes de Millon. Madrid: TEA.

(22) Nardone, G.; Giannotti, E.; Rocchi, R. (2003). Modelos de Familia: conocer y resolver los problemas entre padres e hijos. Barcelona: Herder.

(23) Olsson, I. G.; Nordström, M. L.; Arinell, H.; Von Knorring, A. (1999). Adolescent depression and stressful life event: a case-control study within diagnostic subgroups. Nordic Journal of psychiatry, 53 (5), 339-346.

(24) Oliva, A.; Jiménez, J. M.; Parra, A.; Sánchez-Queija, I. (2008). Acontecimientos vitales estresantes, resiliencia y ajuste adolescente. Revista de Psicopatología y Psicología Clínica, 13 (1), 53-62.

(25) OMS (1993). Tablas de conversión entre la CIE-8, la CIE-9 y la CIE-10. Madrid: Meditor.

(26) PARKer, G. (1992). Early environement. En: E.S. Paykel (Ed.), Handbook of affective disorders, pp. 171-183. New York: Guildford.

(27) Rutter, M.; Thorpe, K.; Greenwood, R.; Smoller, J.W.; Rosembaum, J. F.; Biederman, J.; Kennedy,J.; DAI, D. (1992). Comorbidity of al anxiety in inhibited children. American Journal of Psychiatry, 149 (4), 475-481.

(28) SÁnchez, P.; SAnz, L. J.; Gómez, M. (2007). La psicoterapia de grupo con adolescentes y jóvenes. En: E. Gamo (Ed.), Ante la mirada de los otros. Guía de Terapias Grupales, pp. 59-78. Madrid: Ediciones Libertarias.

(29) Sandler, I. N.; Tein, J: Y.; West S. G. (1994). Coping, stress, and the psychilogical symptoms of children of divorced: a cross-sectional and longitudinal study. Child development, 65, 1744-1763.

(30) Sanz, L. J.; Burgulllo, F.; Escudero, C.; Fernández De La Vega, S.; Jiménez, M. (2006). Características familiares y factores de riesgo en niños y adolescentes con trastornos en Salud Mental. Psicopatología y Salud Mental del niño y del adolescente, 8, 35-44.

(31) Seligman, M. A. (1991). Indefensión. Madrid: Debate.

(32) Sheeber, L. B.; Davis, B.; Leve, C.; Hops, H.; Tildesley, E. L. (2007). Adolescents' relationships with their mothers and fathers: association with depressive disorder and subdiagnostic symptomathology. Journal of abnormal psychology, 116 (1), 144-154.

(33) Siegel, K.; Karus, D.; Raveis, V.H. (1996). Adjustment of children facing the death of a parent due to cancer. Journal of the american acadamy of child and adolescent psychiatry, 35 (4), $442-450$.

(34) Stein, M. B.; Walker, J. R.; Anderson, G.; Hacen, A. L.; Ross, C. A.; Eldridge, G.; Forde, D. R. (1996). Childhood physical and sexual abuse in patients with anxiety disorder in a community sample. American journal of psychiatry, 153, 275-277.

(35) Vélez, C. N.; Johnson, J.; Cohen, P. (1989). A longitudinal analysis of selected risk factors for childhood psychopathology. Journal of the american academy of child and adolescent psychiatry, 28, 861-864.

(36) VILlanueva, C. y García, J. (2000). La especificidad del duelo en la infancia. Psiquiatría Pública, 12 (3), 219-228.

(37) WindLE, M. (1992). A longitudinal study of stress buffering for adolescent problem behaviors. Developmental psychology, 28, 522-530. 
ORIGINALES Y REVISIONES

* Luis Javier Sanz Rodríguez. Psicólogo Clínico. SSM de Parla. Universidad Complutense de Madrid. María Gómez García de la Pedrosa. Psicólogo Clínico. SSM de Parla. María Teresa Almendro Marín. Psicólogo Clínico. SSM de Parla. Candela Rodríguez Campos. PIR Hospital Universitario de Getafe. Ana María Izquierdo Núñez. Trabajadora Social. SSM de Parla. Pilar Sánchez Del Hoyo. Psicólogo Clínico. SSM de Parla.

Correspondencia: Luis Javier Sanz Rodríguez. Servicios de Salud Mental de Parla. C/ Pablo Sorozábal, nº4. Parla (28920). Madrid. Correo electrónico: ljsanz@cop.es

** Recibido: 14/05/2009 